Stabilizability and stability robustness of state derivative feedback controllers

\author{
Wim Michiels \\ Tomáš Vyhlídal \\ Henri Huijberts \\ Henk Nijmeijer
}

Report TW 520, March 2008

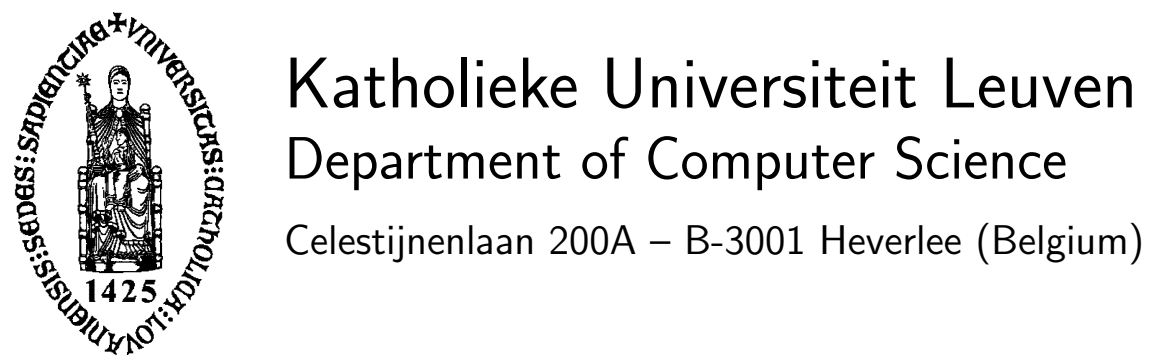




\title{
Stabilizability and stability robustness of state derivative feedback controllers
}

\author{
Wim Michiels \\ Tomáš Vyhlídal \\ Henri Huijberts \\ Henk Nijmeijer
}

Report TW 520, March 2008

Department of Computer Science, K.U.Leuven

\begin{abstract}
We study the stabilizability of a linear controllable system using state derivative feedback control. As a special feature the stabilized system may be fragile, in the sense that arbitrarily small modelling and implementation errors may destroy the asymptotic stability. First, we discuss the pole placement problem and illustrate the fragility of stability with examples of a different nature. We also define a notion of stability, called $p$-stability, which explicitly takes into account the effect of small modelling and implementation errors. Next, we investigate the effect on the fragility of including a low-pass filter in the control loop. Finally, we completely characterize the stabilizability and $p$-stabilizability of linear controllable systems using state derivative feedback. In the stabilizability characterization the odd number limitation, well known in the context of the stabilization of unstable periodic orbits using Pyragas type time-delayed feedback, plays a crucial role.
\end{abstract}

Keywords : stabilizability, feedback, robustness 


\title{
Stabilizability and stability robustness of state derivative feedback controllers
}

\author{
Wim Michiels \\ Department of Computer Science \\ Katholieke Universiteit Leuven \\ Belgium \\ Email: Wim.Michiels@cs.kuleuven.be \\ and \\ Department of Mechanical Engineering \\ Eindhoven University of Technology \\ The Netherlands \\ Tomáš Vyhlídal \\ Centre for Applied Cybernetics \\ Czech Technical University in Prague \\ Czech Republic \\ E-mail: Tomas.Vyhlidal@fs.cvut.cz
}

Henri Huijberts

School of Engineering and Materials Science

Queen Mary, University of London

United Kingdom

Email: H.J.C.Huijberts@qmul.ac.uk

Henk Nijmeijer

Department of Mechanical Engineering

Eindhoven University of Technology

The Netherlands

Email: H.Nijmeijer@tue.nl 


\begin{abstract}
We study the stabilizability of a linear controllable system using state derivative feedback control. As a special feature the stabilized system may be fragile, in the sense that arbitrarily small modelling and implementation errors may destroy the asymptotic stability. First, we discuss the pole placement problem and illustrate the fragility of stability with examples of a different nature. We also define a notion of stability, called $p$-stability, which explicitly takes into account the effect of small modelling and implementation errors. Next, we investigate the effect on the fragility of including a low-pass filter in the control loop. Finally, we completely characterize the stabilizability and $p$-stabilizability of linear controllable systems using state derivative feedback. In the stabilizability characterization the odd number limitation, well known in the context of the stabilization of unstable periodic orbits using Pyragas type time-delayed feedback, plays a crucial role.
\end{abstract}




\section{Introduction}

We analyze the stabilizability and stabilization of the linear or linearized system

$$
\dot{x}(t)=A x(t)+B u(t),
$$

where $x(t) \in \mathbb{R}^{n}$ is the system state vector and $u(t) \in \mathbb{R}^{m}$ is the system input at time $t$, using state derivative feedback,

$$
u(t)=K_{d} \dot{x}(t), \quad K_{d} \in \mathbb{R}^{n \times m} .
$$

In this stabilizability study we explicitly take into account the effects of arbitrarily small modelling, approximation and implementation errors, which may for instance be caused by feedback latency, unmodelled sensor and actuator dynamics and approximations of the derivatives.

From a practical point of view, the motivation for using state derivative feedback (2) instead of conventional state feedback, $u(t)=K_{s} x(t)$, comes from applications where accelerometers are used for measuring the system's motion. Typical applications are in vibration control of mechanical systems where the state variables are positions and velocities, while the accelerations, which are the sensed variables, are directly used for feedback, see $[2,3,1]$. In some applications, including vibration suppression, the fact that the control law (2) keeps the steady state solutions of the uncontrolled system invariant might be considered as a positive feature.

From a theoretical point of view the stabilizability study of the system (1) with the control law (2) is a challenging problem if robustness aspects are taken into account. As will be addressed later in the text, the synthesis of the derivative feedback can be accomplished using modified pole placement methods under a controllability assumption. However, the achieved closed-loop dynamics may be fragile in the sense that the stability of the controlled system may lack robustness against arbitrarily small modelling and implementation errors. In other words, although the nominal system is asymptotically stable, some stability margins may be equal to zero. The main goals of the paper consist of studying this fragility problem and making a complete characterization of the stabilizability of (1) with (2) in the presence of any type of small modelling and implementation errors.

Other problems where a fragility of stability has been observed can be found in the literature. More specifically, a lack of robustness of stability against small feedback delays or against small delay changes has been observed for boundary controlled (hyperbolic) partial differential equations, see e.g. [5, 6, 8, 11, 17, 21], for feedback controlled descriptor systems [10] and for neutral functional differential equations $[12,13]$. In [22] a model for gradient play dynamics is discussed where the discretization of a derivative may induce instability. In $[14,16]$ it was shown that the discretization of distributed delays in control laws, arising in the context of finite spectrum assignment of time-delay systems, may destabilize the system, even if the discretization stepsize is arbitrarily small.

The structure of the paper is as follows: in Section 2 the pole placement problem for the system (1) using the control law (2) is first discussed. Next, a unified framework 
for studying the effect of small modelling and implementation errors is presented and a practical notion of stability is introduced. In Section 3 two examples of a different nature are presented which illustrate that closed-loop stability may not be robust against small modelling and implementation errors. The observations on the instability mechanisms lead us to Section 4, where we investigate to what extent the inclusion of a low-pass filter may solve the robustness problems. Finally, in Sections 5-6 a full characterization of the stabilizability of (1) using (2) is given, with and without taking into account the effect of small modelling and implementation errors. It will be shown that in the former case an important role is played by a condition that boils down to the so-called odd number limitation, well known in the context of the stabilization of unstable periodic solutions using Pyragas type time-delayed controllers [20] and in the context of delay difference feedback [9]. Some concluding remarks end the paper.

The following notation and definitions will be adopted: $\mathbb{C}\left(\mathbb{C}^{+}, \mathbb{C}^{-}\right)$is the set of complex numbers (with strictly positive and strictly negative real parts), and $j=\sqrt{-1}$. For $\lambda \in \mathbb{C}$, $\bar{\lambda}, \Re(\lambda)$ and $\Im(\lambda)$ define the complex conjugate, the real part and the imaginary part of $\lambda$. For $\Omega \subset \mathbb{C}, \partial \Omega$ denotes the boundary of $\Omega$ and $\operatorname{clos}(\Omega)$ its closure. $\mathbb{R}\left(\mathbb{R}^{+}, \mathbb{R}^{-}\right)$denotes the set of real numbers (larger than or equal to zero, smaller than or equal to zero). $\mathbb{N}$ is the set of natural numbers and is assumed to include zero. $\mathbb{Z}$ the set of integers. For an operator or matrix $A, \sigma(A)$ and $\rho(A)$ denote its spectrum and spectral radius respectively. Throughout the paper we make the following assumption.

Assumption 1.1. The pair $(A, B)$ is controllable.

\section{Prerequisites}

\subsection{Pole placement using state derivative feedback}

Note that the closed loop system $(1,2)$ may be rewritten as

$$
\left(I-B K_{d}\right) \dot{x}(t)=A x(t)
$$

Thus, the closed loop system is well-posed if $B K_{d}$ has no eigenvalue equal to one. The characteristic function of the closed loop system is then given by

$$
H_{0}(\lambda):=\operatorname{det}\left(\lambda I-A-B K_{d} \lambda\right)
$$

We first give some preliminary results considering the stability of the closed loop system.

Proposition 2.1. If $\operatorname{det}(A)=0$, then the closed loop system (1,2) has a zero characteristic root for all values of $K_{d}$.

Conditions for arbitrary pole placement by state derivative feedback (2) are given in [1] as follows. 
Proposition 2.2. If the pair $(A, B)$ is controllable, then all characteristic roots of the closed-loop system can be assigned at arbitrarily positions in $\mathbb{C} \backslash\{0\}$ using the control law (2) if and only if $\operatorname{det}(A) \neq 0$.

The following result from [1] establishes relations with pole assignment using state feedback.

Proposition 2.3. Assume that $\operatorname{det}(A) \neq 0$. With the control law (2), the closed-loop system has the same characteristic roots as with the state feedback

$$
u(t)=K_{s} x(t)
$$

if

$$
K_{d}=K_{s}\left(A+B K_{s}\right)^{-1} .
$$

Given the fact that algorithms for designing the controller (5) are widely available, this is an important result. However, algorithms have also been developed for a direct design of the state derivative feedback gain $K_{d}$. A general pole placement technique for state derivative feedback was proposed in [1] for single-input delay free systems and in [2] for multiple-input systems. The same authors proposed an LQR technique for computing state derivative feedback in [3]. The application of acceleration feedback to vibration suppression problems has been discussed at length in [19] and [7].

Remark 2.4. If $\operatorname{det}(-A)<0$, i.e., if the system (1) has an odd number of characteristic roots in $\mathbb{C}^{+}$, stabilization implies that an odd number of unstable roots need to be shifted to the left half plane, while a root cannot cross the imaginary axis at zero. This may sound counter intuitive but is always possible: if $K_{d}$ is increased from zero to the stabilizing value then some characteristic roots move to the right-half plane via infinity, where for the critical value of $K_{d}$ the system is not well-posed. As an example of this one can consider the system

$$
\dot{x}(t)=x(t)+u(t), u(t)=k_{d} \dot{x}(t), \quad u, x \in \mathbb{R}
$$

for which clearly the open loop system has an odd number of characteristic roots in $\mathbb{C}^{+}$. Further, the closed-loop characteristic root $\lambda$ satisfies

$$
\lambda=\frac{1}{1-k_{d}}, \quad k_{d} \neq 1
$$

It is straightforwardly seen that the closed loop system is stable for $k_{d}>1$ and that $\lambda \rightarrow \infty$ if $k_{d} \uparrow 1$. 


\subsection{Framework for robustness analysis}

As we shall illustrate in the next section, the use of state derivative feedback may introduce some fragility, in the sense that arbitrarily small modelling and implementation errors, e.g., arbitrarily small delays in the feedback loop, may change the system's behavior significantly and may even render an asymptotically stable nominal system unstable. To create a unifying framework to study this fragility problem, we assume that the modelling and implementation errors are such that the characteristic function of the actual system is given by:

$$
H(\lambda ; p):=\operatorname{det}\left(\lambda I-\tilde{A}(p)-\tilde{B}(p) G_{1}(\lambda ; p) K_{d} \lambda G_{2}(\lambda ; p)\right),
$$

where $p \in\left(\mathbb{R}^{+}\right)^{n_{p}}$ denotes some parameters and the functions

$$
\begin{aligned}
& \tilde{A}:\left(\mathbb{R}^{+}\right)^{n_{p}} \rightarrow \mathbb{R}^{n \times n}, \quad p \mapsto \tilde{A}(p) ; \\
& \tilde{B}:\left(\mathbb{R}^{+}\right)^{n_{p}} \rightarrow \mathbb{R}^{n \times m}, \quad p \mapsto \tilde{B}(p) ; \\
& G_{1}: \mathbb{C} \times\left(\mathbb{R}^{+}\right)^{n_{p}} \rightarrow \mathbb{C}^{m \times m}, \quad(\lambda, p) \mapsto G_{1}(\lambda ; p), \\
& G_{2}: \mathbb{C} \times\left(\mathbb{R}^{+}\right)^{n_{p}} \rightarrow \mathbb{C}^{n \times n}, \quad(\lambda, p) \mapsto G_{2}(\lambda ; p),
\end{aligned}
$$

satisfy the following assumption.

\section{Assumption 2.5.}

1. The functions $p \mapsto \tilde{A}(p)$ and $p \mapsto \tilde{B}(p)$ are continuous;

2. $\lim _{p \rightarrow 0} \tilde{A}(p)=A ; \lim _{p \rightarrow 0} \tilde{B}(p)=B$;

3. for every $p \in\left(\mathbb{R}^{+}\right)^{n_{p}}$ and $i=1,2$, the functions $G_{i}(\cdot ; p)$ are meromorphic; for every $\lambda \in \mathbb{C}$, the functions $G_{i}(\lambda ; \cdot)$ are continuous;

4. $G_{i}(\lambda ; 0)=I$, for all $\lambda \in \mathbb{C}$ and $i=1,2$;

5. for every compact set $\Omega \subset \mathbb{C}$, we have

$$
\lim _{p \rightarrow 0} \max _{\lambda \in \Omega}\left\|G_{i}(\lambda ; p)-I\right\|=0, i=1,2
$$

6. there exist constants $M, N, P>0$ such that for all $\lambda \in \mathbb{C}$ with $\Re(\lambda) \geq-N$ and for all $p \in\left(\mathbb{R}^{+}\right)^{n_{p}}$ with $\|p\| \leq P$,

$$
\left\|G_{i}(\lambda ; p)\right\| \leq M, i=1,2 .
$$

In Figure 1 a block diagram is drawn of the controlled system in the presence of modelling and implementation errors. For $p=0$, the function (6) reduces to $H_{0}$ in (4). It is clear that $\tilde{A}(p)$ and $\tilde{B}(p)$ model uncertainty on $A$ and $B$. To motivate the inclusion of $G_{1}$ and $G_{2}$ in the control loop we present some examples of a different nature, which all satisfy Assumption 2.5. 


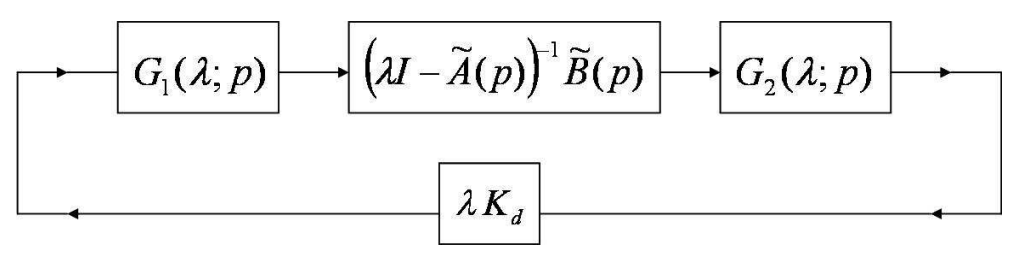

Figure 1: Feedback interpretation of the controlled system.

- Feedback delays. The case where

$$
\begin{aligned}
& n_{p}=n+m, \quad p=\left(\tau_{u_{1}}, \ldots, \tau_{u_{n}}, \tau_{x_{1}}, \ldots, \tau_{x_{n}}\right) \\
& G_{1}(\lambda ; p)=\operatorname{diag}\left(e^{-\lambda \tau_{u_{1}}}, \ldots, e^{-\lambda \tau_{u_{m}}}\right), \quad G_{2}(\lambda, p)=\operatorname{diag}\left(e^{-\lambda \tau_{x_{1}}}, \ldots, e^{-\lambda \tau_{x_{n}}}\right),
\end{aligned}
$$

corresponds to the case where one assumes a time-delay $\tau_{u_{k}}$ in the $k$-th component of the input $u$ and a time-delay $\tau_{x_{l}}$ in the measurement of the $l$-th component of $\dot{x}$, where $1 \leq k \leq m, 1 \leq l \leq n$;

- Numerical computation of derivatives. If, for example,

$$
n_{p}=1, \quad G_{1}(\lambda ; p)=I, \quad G_{2}(\lambda, p)=g_{2}(\lambda ; p) I
$$

with

$$
g_{2}(\lambda ; p)= \begin{cases}\frac{1-e^{-\lambda p}}{\lambda p}, & \lambda p \neq 0 \\ 1, & \lambda p=0\end{cases}
$$

then we get

$$
\lambda K_{d} G_{2}(\lambda ; p)=K_{d} \frac{1-e^{-\lambda p}}{p},
$$

Thus, this corresponds to the situation where $\dot{x}(t)$ in the control law (2) is not measured directly, but computed on-line from the measurements of $x(t)$ using the finite-difference formula

$$
\dot{x}(t) \approx \frac{x(t)-x(t-p)}{p} ;
$$

- Unmodelled dynamics. $G_{1}$ and $G_{2}$ may, for instance, model neglected actuator and sensor dynamics.

From a fragility point of view we are interested in the relationship between the stability properties for $p=0$ and for small $p \neq 0$. For this, we first introduce the following practical notion of closed-loop stability. 
Definition 2.6. The closed-loop system formed by the feedback interconnection of the system (1) with output $x$ and a controller with transfer function $-C(\lambda)$ is $p$-stable if its null solution is asymptotically stable, and for every set of functions (7) satisfying Assumption 2.5 , there is a constant $\hat{p}>0$ such that the zeros of

$$
\operatorname{det}\left(\lambda I-\tilde{A}(p)-\tilde{B}(p) G_{1}(\lambda ; p) C(\lambda) G_{2}(\lambda ; p)\right)
$$

are in $\mathbb{C}^{-}$for all $p \in\left(\mathbb{R}^{+}\right)^{n_{p}}$ with $\|p\|<\hat{p}$.

In this way, requiring that (1), stabilized with (2), be robust against small modelling and implementation errors can be rephrased as requiring the $p$-stability of (1)-(2).

To conclude this section, we note that, for an arbitrary set of functions (7) that satisfy Assumption 2.5, the requirement that the roots of $H_{0}$ in (4) are in $\mathbb{C}^{-}$, along with Assumption 2.5 , is in general not sufficient to guarantee that the zeros of $H$ in $(6)$ are in $\mathbb{C}^{-}$ for sufficiently small $p$. However, an (approximate) matching of $n$ zeros always takes place as follows from the following result.

Proposition 2.7. Assume that $\operatorname{det}\left(I-B K_{d}\right) \neq 0$ and let $\mu_{i} \in \mathbb{C}, 1 \leq i \leq n$, be the eigenvalues of $\left(I-B K_{d}\right)^{-1} A$. There exist a number $\hat{p}>0$ and $n$ continuous functions

$$
\hat{\lambda}_{i}:[0, \hat{p})^{n_{p}} \rightarrow \mathbb{C}, p \mapsto \hat{\lambda}_{i}(p), 1 \leq i \leq n,
$$

which satisfy $H\left(\hat{\lambda}_{i}(p) ; p\right) \equiv 0$ and

$$
\lim _{p \rightarrow 0} \hat{\lambda}_{i}(p)=\mu_{i}, \quad 1 \leq i \leq n .
$$

Proof. Let $\mathcal{B} \subset \mathbb{C}$ be an open disk which contains all zeros of $H_{0}$. The function $H(\lambda ; p)$ uniformly converges on compact subsets of $\mathbb{C}$ to $H_{0}(\lambda)$ as $p \rightarrow 0$. This implies the existence of a number $\hat{p}$ such that

$$
\max _{\lambda \in \partial \mathcal{B}}\left|H_{0}(\lambda)-H(\lambda ; p)\right|<\min _{\lambda \in \partial \mathcal{B}}\left|H_{0}(\lambda)\right|, \quad \forall p \in[0, \hat{p})^{n_{p}} .
$$

By Rouché's Theorem one concludes that $H(\lambda ; p)$ and $H_{0}(\lambda)$ both have $n$ zeros in $\mathcal{B}$ if $p \in[0, \hat{p})^{n_{p}}$. The existence of the functions $\hat{\lambda}_{i}, 1 \leq i \leq n$, follows from the combination of this result with the continuity of the individual zeros of $H$ w.r.t. $p$. The assertion (11) can be shown in a similar way, by letting $\mathcal{B}$ be a disk with arbitrarily small radius centered at a zero of $H_{0}$, and taking the same steps.

In what follows, we focus on the behavior of the other zeros which may be introduced by the implementation or approximation. 


\section{Sensitivity of stability to arbitrarily small model- ling and implementation errors}

With two case studies we first show that even if all zeros of (4) are in $\mathbb{C}^{-}$, then (6) may have zeros in $\mathbb{C}^{+}$for arbitrarily small values of $p$. The first case study, inspired by [22], concerns a numerical approximation of the derivatives in the control law, the second concerns the effect of a neglected time-delay. In both cases the eigenvalue distribution of $B K_{d}$ determines the position of the characteristic roots introduced by the approximation.

\subsection{Approximation of derivatives}

With the approximation (10) of the derivatives in the control law (2), or, equivalently, with the transfer functions (9), the characteristic function of the closed-loop system becomes:

$$
I_{1}(\lambda ; p):=\operatorname{det}\left(\lambda I-A-B K_{d} \frac{1-e^{-\lambda p}}{p}\right) .
$$

We then have the following result.

Proposition 3.1. If $B K_{d}$ has at least one eigenvalue which does not belong to $\operatorname{clos}(S)$, where

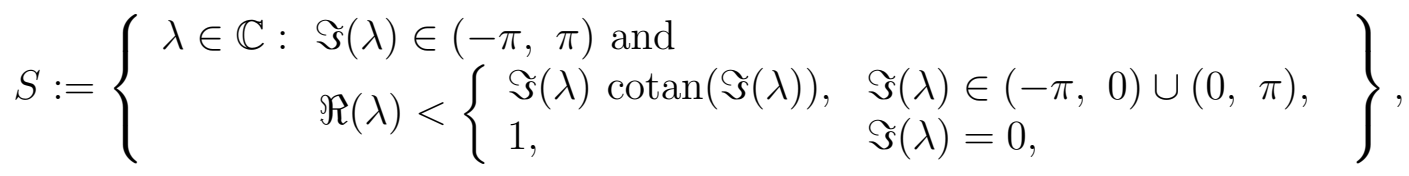

then there exist numbers $\hat{p}>0, c>0$ and a function $\hat{\lambda}:(0, \hat{p}) \rightarrow \mathbb{C}, p \mapsto \hat{\lambda}(p)$, such that

$$
I_{1}(\hat{\lambda}(p) ; p)=0, \text { and } \Re(\hat{\lambda}(p))>\frac{c}{p}
$$

for all $p \in(0, \hat{p})$.

If all eigenvalues of $B K_{d}$ belong to the set $S$, then there exist numbers $\hat{p}>0$ and $c>0$ such that for all $0<p \leq \hat{p}$, the function $I_{1}(\lambda ; p)$ has exactly $n$ zeros in the half plane $\left\{\lambda \in \mathbb{C}: \Re(\lambda)>-\frac{c}{p}\right\}$.

Proof. Let

$$
G(\lambda ; p):=p^{n} I_{1}\left(\frac{\lambda}{p} ; p\right)=\operatorname{det}\left(\lambda I-A p-B K_{d}\left(1-e^{-\lambda}\right)\right)
$$

and let

$$
\tilde{G}(\lambda)=\operatorname{det}\left(\lambda I-B K_{d}\left(1-e^{-\lambda}\right)\right) .
$$

It is clear that

$$
\tilde{G}(\lambda)=0 \Longleftrightarrow \lambda-\lambda_{i}\left(1-e^{-\lambda}\right)=0, i=1, \ldots, n,
$$


where $\lambda_{i}, i=1, \ldots, m$, are the eigenvalues of $B K_{d}$. In what follows we distinguish the following two cases.

Case 1: $\exists k \in\{1, \ldots, n\}: \lambda_{k} \notin \operatorname{clos}(S)$. Following from expression (13) and Lemma A.1, $\tilde{G}$ has a zero in $\mathbb{C}^{+}$. By the uniform convergence of $G(\cdot ; p)$ to $\tilde{G}$ on compact sets as $p \rightarrow 0$ and Rouché's Theorem, there exist constants $c_{1}>0$ and $\hat{p}_{1}>0$ such that for all $p \in\left(0, \hat{p}_{1}\right), G(\cdot ; p)$ has a zero in the right half plane $\left\{\lambda \in \mathbb{C}: \Re(\lambda)>c_{1}\right\}$. Hence by $(15)$, $I_{1}(\cdot ; p)$ has a zero in the half plane $\left\{\lambda \in \mathbb{C}: \lambda>c_{1} / p\right\}$.

Case 2: $\lambda_{i} \in S, 1 \leq i \leq n$. By expression (13) and Lemma A.1 from the appendix, $\tilde{G}$ has a zero at the origin with multiplicity $n$ while all other zeros are confined to the open left half plane. Since the zeros of $G(\cdot ; p)$ belong to the set

$$
\left\{\lambda \in \mathbb{C}:|\lambda| \leq\|A p\|+\left\|B K_{d}\right\|\left(1+e^{-\Re(\lambda)}\right)\right\}
$$

and $G(\cdot ; p)$ uniformly converges to $\tilde{G}$ on compact sets as $p \rightarrow 0$, an application of Rouché's Theorem allows to conclude the existence of constants $c>0$ and $\hat{p}>0$ such that for all $p \in(0, \hat{p}):$

1. $G(\cdot ; p)$ has exactly $n$ zeros in the half plane $\{\lambda \in \mathbb{C}: \Re(\lambda)>-c\}$;

2. all other zeros of $G(\cdot ; p)$ are in the half plane $\{\lambda \in \mathbb{C}: \Re(\lambda)<-c\}$.

This is equivalent to the assertion of the proposition when taking into account the equivalence

$$
G(\lambda ; p, 0)=0 \Longleftrightarrow I_{1}(\lambda p ; p)=0 .
$$

Remark 3.2. In [22] the special case is treated where $A$ and $B K_{d}$ are multiples of each other, which stems from a gradient play dynamics application.

Corollary 3.3. If $B K_{d}$ has an eigenvalue outside $\operatorname{clos}(S)$ and the control law (2) is stabilizing, then the closed-loop system is not p-stable. Furthermore, $I_{1}(\lambda ; p)$ has zeros in $\mathbb{C}^{+}$ for arbitrarily small values of $p$.

\subsection{Feedback delay}

We assume the presence of an unmodelled feedback delay $p$ in all input channels, that is,

$$
G_{1}(\lambda ; p)=e^{-\lambda p} I, \quad G_{2}(\lambda ; p)=I .
$$

Then the characteristic function becomes.

$$
I_{2}(\lambda ; p):=\operatorname{det}\left(\lambda I-A-B K_{d} \lambda e^{-\lambda p}\right) .
$$

As shown in the following result, the eigenvalue distribution of $B K_{d}$ determines the behavior of the zeros thus introduced. 
Proposition 3.4. If $\rho\left(B K_{d}\right)>1$, there exist numbers $\hat{p}>0, c>0$ and a function $\hat{\lambda}:(0, \hat{p}) \rightarrow \mathbb{C}, p \mapsto \hat{\lambda}(p)$, such that

$$
I_{2}(\hat{\lambda}(p) ; p)=0, \text { and } \Re(\hat{\lambda}(p))>\frac{c}{p}
$$

for all $p \in(0, \hat{p})$.

If $\rho\left(B K_{d}\right)<1$, there exist numbers $\hat{p}>0$ and $c>0$ such that for all $0<p \leq \hat{p}$, the function $I_{2}(\lambda ; p)$ has exactly $n$ zeros in the half plane $\left\{\lambda \in \mathbb{C}: \Re(\lambda)>-\frac{c}{p}\right\}$.

Proof. If $\rho\left(B K_{d}\right)>1$, it follows from the theory developed in $[4,15]$ that for all $p>0$, there exists a sequence of complex numbers $\left\{\lambda_{\nu}\right\}_{\nu \geq 0}$ such that

$$
\begin{aligned}
& I_{2}\left(\lambda_{\nu} ; p\right)=0, \nu \geq 1, \\
& \lim _{\nu \rightarrow \infty} \Im\left(\lambda_{\nu}\right)=+\infty, \quad \lim _{\nu \rightarrow \infty} \Re\left(\lambda_{\nu}\right)=\frac{\log \left(\rho\left(B K_{d}\right)\right)}{p} .
\end{aligned}
$$

By letting $c=\left(\log \left(\rho\left(B K_{d}\right)\right)\right) / 2$ the statement of the proposition follows.

If $\rho\left(B K_{d}\right)<1$, there exists a $c>0$ such that $\rho\left(B K_{d} e^{c}\right)<1$. Next, we let $\lambda_{0}$ be a zero of $I_{2}(\cdot ; p)$ satisfying $\Re(\lambda)>-c / p$. As the matrix $\left(I-B K_{d} e^{-\lambda p}\right)$ is invertible if $\Re(\lambda)>-c / p$, we get

$$
\operatorname{det}\left(\lambda_{0} I-\left(I-B K_{d} e^{-\lambda_{0} p}\right)^{-1} A\right)=0 .
$$

This implies

$$
\left|\lambda_{0}\right| \leq \max _{\Re(\lambda) \geq-c / p}\left\|\left(I-B K_{d} e^{-\lambda p}\right)^{-1} A\right\| \leq M,
$$

where

$$
M:=\max _{\Re(\lambda) \geq-c}\left\|\left(I-B K_{d} e^{-\lambda}\right)^{-1} A\right\| .
$$

Consequently, all zeros of $I_{2}(\cdot ; p)$ in the half plane $\{\lambda \in \mathbb{C}: \Re(\lambda) \geq-c / p\}$ also lie in the disk $\{\lambda \in \mathbb{C}:\|\lambda\| \leq M\}$. Combining this result with Proposition 2.7 yields the assertion to be proven.

Corollary 3.5. If $B K_{d}$ has an eigenvalue outside the unit disk and the control law (2) is stabilizing, then the closed-loop system is not practically stable. Furthermore, $I_{2}(\lambda ; p)$ has zeros in $\mathbb{C}^{+}$for arbitrarily small values of $p$.

\section{Filtered state derivative feedback}

Using Assumption 2.5 and Rouché type arguments, all but $n$ zeros of $H(\lambda ; p)$ move off to infinity as the parameter $p$ tends to zero. An obstruction to $p$-stability occurs if some of these zeros move off to infinity without leaving the closed right half plane. Such situations are illustrated with Proposition 3.1 and Proposition 3.4. A natural way to prevent the presence of zeros with a large modulus in the right half plane consists of including a lowpass filter in the control scheme. Note that such an approach has already been successfully 
applied to the discretization of distributed delay controllers in the context of finite spectrum assignment, where similar robustness problems occur $[14,16]$.

When applying a first order filter to the control law (2), the controller becomes:

$$
T \dot{u}(t)+u(t)=K_{d} \dot{x}(t)
$$

where $T=1 / \omega_{f}$ is the time constant of the filter, and $\omega_{f}$ is its cutoff frequency.

The feedback system that consists of (1) and (17) is given by

$$
\dot{z}(t)=\left[\begin{array}{cc}
A & B \\
0 & -\frac{1}{T} I
\end{array}\right] z(t)+\left[\begin{array}{cc}
0 & 0 \\
\frac{1}{T} K_{d} & 0
\end{array}\right] \dot{z}(t),
$$

where $z(t)=\left[\begin{array}{ll}x(t)^{T} & u(t)^{T}\end{array}\right]^{T}$. This system can be rewritten as

$$
\dot{z}(t)=\left[\begin{array}{cc}
A & B \\
\frac{1}{T} K_{d} A & \frac{1}{T}\left(K_{d} B-I\right)
\end{array}\right] z(t) .
$$

Let $J_{0}(\lambda ; T)$ be the characteristic function of $(18,19)$, that is,

$$
J_{0}(\lambda ; T):=\operatorname{det}\left(\lambda I-\left[\begin{array}{cc}
A & B \\
\frac{1}{T} K_{d} A & \frac{1}{T}\left(K_{d} B-I\right)
\end{array}\right]\right) .
$$

We perform the subsequent analysis in two steps. First, we discuss the effect of the introduction of the filter on the stability of the nominal system. Next, we investigate the effect of small approximation and implementation errors. We end the section with a brief discussion of the results.

\subsection{Filter design}

Intuitively, one might expect that the introduction of a filter with a sufficiently high cut-off frequency (i.e., $T$ is sufficiently small) has little influence on the dynamic behavior of the nominal system. However, this is not always the case, as follows from the following result.

Proposition 4.1. Assume that $\operatorname{det}\left(I-B K_{d}\right) \neq 0$ and let $\xi_{i} \in \mathbb{C}, 1 \leq i \leq n$, be the eigenvalues of $\left(I-B K_{d}\right)^{-1} A$. There exist a number $\hat{T}$ and $n$ continuous functions

$$
\hat{\eta}_{i}:(0, \hat{T}) \rightarrow \mathbb{C}, T \mapsto \hat{\eta}_{i}(T), 1 \leq i \leq n,
$$

which satisfy $J\left(\hat{\eta}_{i}(T) ; T\right) \equiv 0$ and

$$
\lim _{T \rightarrow 0+} \hat{\eta}_{i}(T)=\xi_{i}, \quad 1 \leq i \leq n .
$$

If $\left(K_{d} B-I\right)$ is Hurwitz, then there exist numbers $\hat{c}>0, \hat{T}>0$ such that for all $T \in(0, \hat{T})$, the function $J_{0}(\lambda ; T)$ has exactly $n$ zeros in the half plane

$$
\left\{\lambda \in \mathbb{C}: \Re(\lambda)>-\frac{\hat{c}}{T}\right\} .
$$


If $\left(K_{d} B-I\right)$ has an eigenvalue in the open right half plane then there exist numbers $\hat{c}>0$ and $\hat{T}>0$ such that for all $T \in(0, \hat{T})$, the function $J_{0}(\lambda ; T)$ has a zero in the half plane

$$
\left\{\lambda \in \mathbb{C}: \Re(\lambda)>\frac{\hat{c}}{T}\right\}
$$

Proof. The proof follows the same steps as the proofs of Propositions 2.7 and 3.1, and it is therefore not developed in detail. We restrict ourselves to some consideration on the large eigenvalues introduced by the filter. From the normalized characteristic function

$$
T^{n+m} J_{0}\left(\frac{\mu}{T} ; T\right)=\operatorname{det}\left(\mu I-\left[\begin{array}{cc}
A T & B T \\
K_{d} A & \left(K_{d} B-I\right)
\end{array}\right]\right)
$$

where the argument of $\operatorname{det}(\cdot)$ becomes triangular as $T \rightarrow 0+$, it is apparent that for small values of $T$, the eigenvalues of $\left(K_{d} B-I\right)$ determine the large eigenvalues of the closed-loop system.

From Proposition 4.1 one concludes that the presence of the filter may induce an additional stability constraint, namely the Hurwitz stability of the matrix $\left(K_{d} B-I\right)$.

Remark 4.2. The above results can also be interpreted in terms of robustness of the unfiltered feedback system (1)-(2). If the nominal system (1)-(2) is asymptotically stable but $\left(K_{d} B-I\right)$ is not Hurwitz, then the stability of the nominal system is not robust against arbitrarily small modelling error described by

$$
n_{p}=1 ; G_{1}(\lambda ; p)=I ; \quad G_{2}(\lambda ; p)=\frac{1}{1+p \lambda} .
$$

Note that (22) satisfies Assumption 2.5. In this way the above analysis can be seen as another illustration of the fragility, already illustrated in the previous section with two examples of a different nature.

\subsection{Effect of small modelling and implementation errors}

We consider the controller (17) and assume that $T$ and $K_{d}$ are such that the null solution of (18) is asymptotically stable.

In the presence of small modelling and approximation errors as described in Section 2.1 the characteristic function (20) becomes:

$$
\begin{aligned}
& J(\lambda ; T, p):= \\
& \operatorname{det}\left(\lambda I-\left[\begin{array}{cc}
\tilde{A}(p) & \tilde{B}(p) \\
\frac{1}{T} G_{1}(\lambda ; p) K_{d} G_{2}(\lambda ; p) \tilde{A}(p) & \frac{1}{T}\left(G_{1}(\lambda ; p) K_{d} G_{2}(\lambda ; p) \tilde{B}(p)-I\right)
\end{array}\right]\right),
\end{aligned}
$$

where $\tilde{A}, \tilde{B}, G_{1}, G_{2}$ satisfy Assumption 2.5. The corresponding block diagram of the closedloop system is displayed in Figure 2.

Due to the low-pass filter in the control loop, the closed-loop system (1) and (17) is $p$-stable in the sense of Definition 2.6, as can be concluded from the following proposition. 


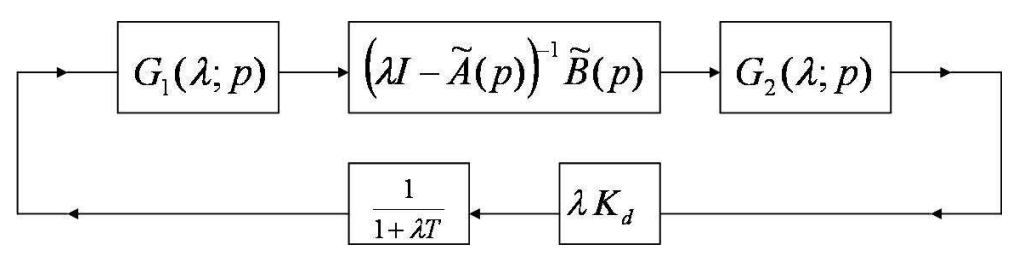

Figure 2: Feedback interpretation of the controlled system with a low-pass filter included in the feedback.

Proposition 4.3. Assume that $T>0$ is fixed. Let $\xi_{i} \in \mathbb{C}, 1 \leq i \leq n+m$, be the zeros of $J_{0}(\lambda ; T)$. There exist a number $\hat{p}$ and $n+m$ continuous functions

$$
\hat{\eta}_{i}:[0, \hat{p})^{n_{p}} \rightarrow \mathbb{C}, p \mapsto \hat{\eta}_{i}(p), 1 \leq i \leq n+m,
$$

which satisfy $J\left(\hat{\eta}_{i}(p) ; T, p\right) \equiv 0$ and

$$
\lim _{p \rightarrow 0} \hat{\eta}_{i}(p)=\xi_{i}, \quad 1 \leq i \leq n+m .
$$

Furthermore, there exist numbers $\tilde{p}>0$ and $c>0$ such that for all $p \in\left(\mathbb{R}^{+}\right)^{n_{p}}$ with $\|p\| \leq \tilde{p}$, the functions $J(\lambda ; T, p)$ and $J_{0}(\lambda ; T)$ have the same number of zeros in the half plane

$$
\{\lambda \in \mathbb{C}: \Re(\lambda) \geq-c\} .
$$

Proof. The proof of the first assertion is similar to the proof of Proposition 2.7, and is omitted. For the second assertion, choose numbers $N$ and $P$ according to Item 6 . of Assumption 2.5. Choose $c \in(0, N)$ such that $J_{0}$ has no zero with real part equal to $c$. Let $\lambda_{0}$ be a zero of $J\left(\cdot ; T, p_{0}\right)$ where $\left\|p_{0}\right\|<P$. If $\Re\left(\lambda_{0}\right) \geq-c$, then we get from (23):

$$
\lambda_{0} \in \sigma\left(\left[\begin{array}{cc}
\tilde{A}(p) & \tilde{B}(p) \\
\frac{1}{T} G_{1}\left(\lambda_{0} ; p\right) K_{d} G_{2}\left(\lambda_{0} ; p\right) \tilde{A}(p) & \frac{1}{T}\left(G_{1}\left(\lambda_{0} ; p\right) K_{d} G_{2}\left(\lambda_{0} ; p\right) \tilde{B}(p)-I\right)
\end{array}\right]\right) .
$$

As $\rho(\cdot) \leq\|\cdot\|$, this implies that $\left|\lambda_{0}\right| \leq R$, where

$$
\begin{aligned}
& R= \\
& \sup \left\{\left\|\left[\begin{array}{cc}
\tilde{A}(p) & \tilde{B}(p) \\
\frac{1}{T} G_{1}(\lambda ; p) K_{d} G_{2}(\lambda ; p) \tilde{A}(p) & \frac{1}{T}\left(G_{1}(\lambda ; p) K_{d} G_{2}(\lambda ; p) \tilde{B}(p)-I\right)
\end{array}\right]\right\|:\right. \\
& \left.\lambda \in \mathbb{C}, p \in \mathbb{R}^{n_{p}}, \Re(\lambda) \geq-c,\|p\| \leq P\right\} .
\end{aligned}
$$

Note that by Assumption 2.5 the right hand side of (26) is finite. We conclude that if $\|p\| \leq P$, then all zeros of $J(\cdot ; T, p)$ in the half plane $(25)$ are confined to the compact set

$$
\Omega:=\{\lambda \in \mathbb{C}: \Re(\lambda) \geq-c,|\lambda| \leq R\} .
$$


Next, from Assumption 2.5 it follows that $J(\lambda ; T, p)$ converges to $J_{0}(\lambda ; T)$ uniformly on compact sets as $p \rightarrow 0$. Hence, we can choose $\tilde{p} \in(0, P)$ such that for all $p \in \mathbb{R}^{n_{p}}$ with $\|p\| \leq \tilde{p}$ the following estimate holds:

$$
\max _{\lambda \in \partial \Omega}\left|J_{0}(\lambda ; T)-J(\lambda ; T, p)\right| \leq \min _{\lambda \in \partial \Omega}\left|J_{0}(\lambda ; T)\right| .
$$

The second assertion of the proposition then follows from an application of Rouché's Theorem.

\subsection{Discussion}

In Section 3 we have shown by construction that (unfiltered) state derivative feedback may lead to a fragile closed-loop system in the sense that the closed-loop system is stable but not $p$-stable. This was illustrated for a numerical approximation of derivatives by a finite difference formula and for a small delay in the feedback loop. In both cases the eigenvalues of the matrix $B K_{d}$ determine the robustness of stability. However, even if the resulting conditions on the gain $K_{d}$ are satisfied, stability may still lack robustness against other type perturbations satisfying Assumption 2.5.

In this section we have shown that if the filtered derivative feedback control law (17) is stabilizing, then the closed-loop system is $p$-stable. However, the existence of a stabilizing filtered derivative feedback for the nominal system may again impose restrictions on the gain, as expressed in Proposition 4.1. Summarizing, we have the following result:

Theorem 4.4. Assume that the control law (2) asymptotically stabilizes the system (1). If the matrix $\left(B K_{d}-I\right)$ is Hurwitz, then the filtered control law (17) is stabilizing for small values of $T$ and results in a p-stable closed-loop system.

If $\left(B K_{d}-I\right)$ has an eigenvalue in $\mathbb{C}^{+}$, then the closed-loop system with the control law (2) is not p-stable. Furthermore, the filtered control law (17) is not stabilizing for small values of $T$.

Proof. Follows from Propositions 4.1, Remark 4.2 and Proposition 4.3.

Further refinements will be made in the next section, where relations between stabilizing values of $K_{d}$ and the eigenvalue distribution of $\left(B K_{d}-I\right)$ will be taken into account.

\section{Conditions for $p$-stabilizability}

In this section we discuss the $p$-stabilizability problem and the design of stabilizing state derivative controllers of the form (2) or (17). As we shall see, the condition $\operatorname{det}(-A)>0$, which is satisfied if $A$ has no zero eigenvalue and an even number of eigenvalues in the closed right half plane, will play a crucial role.

Throughout this section we assume that $(A, B)$ is controllable and that $A$ is cyclic. Remarks on the non-cyclic case will be made in the next section.

We start by stating a technical lemma. 
Lemma 5.1. Assume that $\operatorname{det}(-A)<0$. If the control law (2) is stabilizing, then the matrix $B K_{d}$ has a real eigenvalue larger than one.

Proof. The proof is based on a continuation argument. If we consider the feedback $u=$ $k K_{d} \dot{x}(t)$, where $k \in[0,1]$ is a real parameter, then the closed loop system becomes

$$
\left(I-k B K_{d}\right) \dot{x}(t)=A x(t) .
$$

Since $\operatorname{det}(-A)<0$, for $k=0$ the system has an odd number of characteristic roots in the open right half plane. On the other hand, for $k=1$ it has an even number of characteristic roots in the open right half plane (zero) as it is assumed that (2) is stabilizing. Because the characteristic roots appear in complex conjugate pairs and depend continuously on $k$, there must be a value of $k=\tilde{k} \in(0,1)$ for which there is a characteristic root either at zero, or "at infinity". The former is not possible because a zero characteristic root is invariant w.r.t. changes of $k$ and would contradict the stability for $k=1$. The latter implies that $\operatorname{det}\left(I-\tilde{k} B K_{d}\right)=0$. Consequently, $\tilde{k} B K_{d}$ has an eigenvalues equal to one, or, equivalently, $B K_{d}$ has a real eigenvalue equal to $1 / \tilde{k}>1$.

The two following theorems are direct corollaries.

Theorem 5.2. If $\operatorname{det}(-A)<0$ and the control law (2) is stabilizing, then the closed-loop system is not $p$-stable.

Proof. This result can be shown in three different ways. As $B K_{d}$ has an eigenvalue larger than one, an approximation of the derivative with a finite difference scheme (Proposition 3.1), a small feedback delay (Proposition 3.4), as well as a neglected first order lag (Proposition 4.1 - Remark 4.2) destroy stability.

Theorem 5.3. If $\operatorname{det}(-A)<0$, then the system cannot be stabilized with a control law of the form (17). Moreover, every dynamic control law of the form

$$
\dot{\zeta}(t)=A_{f} \zeta(t)+B_{f} \dot{x}(t), u(t)=C_{f} \zeta(t),
$$

with $A_{f}$ Hurwitz, results in an unstable closed-loop system.

Proof. The first assertion is a consequence of Theorem 4.4 and Lemma 5.1. The proof of the second assertion is by contradiction and employs a continuation argument. Assume that the control law (28) is stabilizing. With the parameterized control law

$$
\dot{\zeta}(t)=A_{f} \zeta(t)+k B_{f} \dot{x}(t), u(t)=C_{f} \zeta(t),
$$

with parameter $k \in[0,1]$, the closed loop system becomes

$$
\left(I-\left[\begin{array}{cc}
0 & 0 \\
k B_{f} & 0
\end{array}\right]\right) \dot{\xi}(t)=\left[\begin{array}{cc}
A & B C_{f} \\
0 & A_{f}
\end{array}\right] \xi(t),
$$

where $\xi(t)=\left[x(t)^{T} \quad \zeta^{T}(t)\right]^{T}$. For $k=0$ the system has an odd number of characteristic roots in the open right half plane as $\operatorname{det}(-A) \operatorname{det}\left(-A_{f}\right)<0$, while for $k=1$ it has an even number of characteristic roots in the open right half plane (zero) as it is assumed that 
(28) is stabilizing. Because the characteristic roots appear in complex conjugate pairs and depend continuously on $k$, there must be a value of $k=\tilde{k} \in(0,1)$ for which there is a characteristic root either at zero, or "at infinity". The former is not possible because a characteristic root at zero is invariant w.r.t. changes of $k$, which contradicts the stability for $k=1$, the latter is not possible because the matrix

$$
I-\left[\begin{array}{cc}
0 & 0 \\
k B_{f} & 0
\end{array}\right]
$$

is invertible for all values of $k$.

Remark 5.4. This result is expected from the stabilization mechanism outlined in Remark 2.4 and the fact that a filter prevents characteristic roots with a large modulus.

Next, we consider the case where $\operatorname{det}(-A)>0$. We first have the following lemma.

Lemma 5.5. If $\operatorname{det}(-A)>0$, then there always exists a stabilizing control law of the form (2) for which all eigenvalues of $B K_{d}$ are zero.

Proof. The existence of such a control law is shown by construction in the proof of Theorem 2 of [9]. To make this paper self contained, we outline the main steps.

There exist a transformation of the state, $z=T_{x} x$, and of the input, $w=T_{u} u$, that put the system (1) in the controller canonical form

$$
\dot{z}(t)=A_{c} z(t)+B_{c} w(t)
$$

with

$$
A_{c}=\left[\begin{array}{ccccc}
0 & 1 & 0 & \cdots & 0 \\
0 & 0 & 1 & \cdots & 0 \\
\vdots & & \ddots & \ddots & \vdots \\
0 & & & 0 & 1 \\
-a_{n} & \cdots & \cdots & -a_{2} & -a_{1}
\end{array}\right], \quad B_{c}=\left[\begin{array}{c|c|c|c}
0 & & & \\
\vdots & & & \\
\vdots & \tilde{B}_{2} & \cdots & \tilde{B}_{m} \\
0 & & & \\
1 & & &
\end{array}\right]
$$

The control law

$$
w(t)=\tilde{K}_{d} \dot{z}(t)
$$

where

$$
\tilde{K}_{d}=\left[\begin{array}{cccc}
-k_{n-1} & \cdots & -k_{1} & 0 \\
0 & \cdots & \cdots & 0 \\
\vdots & & & \vdots \\
0 & \cdots & \cdots & 0
\end{array}\right],
$$

then results in a closed-loop system with characteristic equation

$$
\lambda^{n}+\sum_{l=1}^{n-1}\left(a_{l}+k_{l}\right) \lambda^{n-l}+a_{n}=0 .
$$


As $a_{n}=\operatorname{det}(-A)>0$, there always exist stabilizing values of $k_{1}, \ldots, k_{n-1}$. Furthermore, all eigenvalues of $B_{c} \tilde{K}_{d}$ are equal to zero. In the original coordinates the control law (31) becomes:

$$
u(t)=T_{u}^{-1} \tilde{K}_{d} T_{x} \dot{x}(t):=K_{d} \dot{x}(t) .
$$

By combining Lemma 5.5 with Theorem 4.4 we arrive at the following result.

Theorem 5.6. If $\operatorname{det}(-A)>0$, then there always exists a stabilizing controller of the form (17) for which the closed-loop system is p-stable.

The obtained stabilizability conditions are summarized in Table 1.

\begin{tabular}{|l||l|l|l|}
\hline & $\operatorname{det}(-A)<0$ & $\operatorname{det}(-A)=0$ & $\operatorname{det}(-A)>0$ \\
\hline \hline stabilizable & yes & no & yes \\
\hline$p$-stabilizable & $\begin{array}{l}\text { no } \\
\text { (neither with }(2) \text { nor with }(17) \text { and }(28))\end{array}$ & no & $\begin{array}{l}\text { yes } \\
(\text { with }(17))\end{array}$ \\
\hline
\end{tabular}

Table 1: $p$-stabilizability of the controllable system (1) using state derivative feedback. $A$ is assumed cyclic.

\section{Remarks on the non-cyclic case}

If the cyclic index ${ }^{1}$ of $A$ is $k>1$, then there always exist transformations of the state, $z=T_{x} x$ and the input, $w=T_{u} u$, which transform (1) into

$$
\dot{z}(t)=A_{c} z(t)+B_{c} w(t),
$$

where

$$
A_{c}=\left[\begin{array}{ccc}
A_{11} & & 0 \\
& \ddots & \\
0 & & A_{k k}
\end{array}\right], B_{c}=\left[\begin{array}{ccc|cc}
B_{11} & \cdots & B_{1 k} & \ldots & B_{1 m} \\
& \ddots & \vdots & & \vdots \\
0 & & B_{k k} & \ldots & B_{k m}
\end{array}\right],
$$

with $A_{i i}$ cyclic and the pair $\left(A_{i i}, B_{i i}\right)$ controllable for each $i=1, \ldots, k$.

As their proofs do not depend on the cyclic index of $A$, Theorems 5.2-5.3 remain valid.

Theorem 6.1. If $\operatorname{det}(-A)<0$ and the control law (2) is stabilizing, then the closed-loop system is not p-stable. Furthermore, every dynamic control law of the form (28), with $A_{f}$ Hurwitz, results in an unstable closed-loop system.

\footnotetext{
${ }^{1}$ the maximum of the geometric multiplicities of its eigenvalues
} 
Based on a decomposition into $k$ sub-problems induced by the canonical form (32), and an application of Theorem 5.6 to each sub-problem, we arrive at the following result.

Theorem 6.2. If $\operatorname{det}\left(-A_{i i}\right)>0,1 \leq i \leq k$, then there always exists a stabilizing controller of the form (17) for which the closed-loop system is p-stable.

When comparing Theorem 6.1 with Theorem 6.2 , the stabilizability analysis is complete if one can make an assertion for the case where

$$
\operatorname{det}(-A)>0, \quad \exists i \in\{1, \ldots, k\}: \operatorname{det}\left(-A_{i i}\right)<0 .
$$

This is still an open problem. The following example indicates that in such case a lack of practical stability may occur.

Example 6.3. The system

$$
\left\{\begin{array}{l}
\dot{x}_{1}(t)=x_{1}(t)+u_{1}(t) \\
\dot{x}_{2}(t)=x_{2}(t)+u_{2}(t)
\end{array}\right.
$$

is of the form (32) with $A_{c}=I, B_{c}=I$ and $A_{11}=A_{22}=1$. Clearly, we have $\operatorname{det}\left(-A_{c}\right)>0$, but $\operatorname{det}\left(-A_{11}\right)=\operatorname{det}\left(-A_{22}\right)<0$.

The control law

$$
u(t)=K_{d} \dot{x}(t), K_{d} \in \mathbb{R}^{2 \times 2},
$$

is stabilizing if and only if the eigenvalues of the matrix $K_{d}$ have real part larger one. By both Proposition 3.1 and Proposition 3.4, the closed-loop system is not $p$-stable.

If we apply the filtered control law

$$
T \dot{u}(t)+u(t)=K_{d} \dot{x}(t), T>0, K_{d} \in \mathbb{R}^{2 \times 2},
$$

then the characteristic equation of the closed-loop is given by

$$
\operatorname{det}\left(\lambda^{2} I+\lambda\left(\frac{1}{T}\left(I-K_{d}\right)-I\right)-\frac{1}{T} I\right)=0,
$$

As this equation has a zero in $\mathbb{C}^{+}$for all values of $T$ and $K_{d}$, stabilization is not possible.

\section{Concluding remarks}

The possible lack of stability robustness of the stabilized system (1)-(2) against arbitrarily small modelling and implementation errors, which was illustrated with several example case-studies, led us to the practical notion of $p$-stability. The stabilizability and $p$-stabilizability of the system (1) with the (filtered) control law (2) was characterized.

For the generic case where $A$ is cyclic, a complete characterization of stabilizability is described in Table 1. Surprisingly, if $\operatorname{det}(-A)<0$, which is satisfied if $A$ has an odd 
number of eigenvalues in the open right half plane, the system is stabilizable, but not pstabilizable, neither using static feedback nor using dynamic feedback. This shows that the so-called odd number condition, $\operatorname{det}(-A)<0$, well known in the context of Pyragas type time-delayed feedback controllers, is also a fundamental obstruction to stabilizability in this context, yet only appears at a second stage, where robustness aspects are considered.

For the case where $A$ is non-cyclic a full characterization of stabilizability and $p$ stabilizability was made, except when (33) holds, that is, in the canonical form there are subsystems which satisfy the odd number condition, but the whole systems does not. Example 6.3 illustrates that again a lack of robustness of stability may occur and suggests that the necessary and sufficient $p$-stabilizability condition in the non-cyclic case is given by $\operatorname{det}\left(-A_{i i}\right)>0$, for all $i=1, \ldots, k$. Whether or not this is true is an open problem and a topic of further research.

Motivated by the large number of applications of sampled-data systems, which appear, for instance, in network controlled systems, it is also worthwhile to investigate to what extent the controller construction, observed phenomena and robustness issues discussed in the paper carry over to the hybrid case where the controller is discrete and difference based. In the overview article [14], where one investigates the effect on stability of approximating distributed delays in a class of control laws for time-delay systems, it is shown that also with pure discrete approximations of control laws fragility problems may occur, and may lead to phenomena which are different than those observed for continuous approximations.

\section{Acknowledgements}

This paper present results of the Belgian Programme on Interuniversity Poles of Attraction, initiated by the Belgian State, Prime Ministers Office for Science, Technology and Culture, and of the Center of Excellence on Optimization in Engineering of the K.U.Leuven. Wim Michiels is a postdoctoral fellow of the Fund for Scientific Research -Flanders (Belgium). The research has been supported by the Ministry of Education of the Czech Republic under the Project $1 \mathrm{M} 0567$.

\section{References}

[1] T.H.S Abdelaziz and M. Valášek. Pole-placement for siso linear systems by statederivative feedback. IEE Proceedings - Control Theory and Applications, 151:377-385, 2004.

[2] T.H.S. Abdelaziz and M. Valášek. Direct algorithm for pole placement by statederivative feedback for mult-input linear systems - nonsingular case. Kybernetika, 41:637-660, 2005.

[3] T.H.S Abdelaziz and M. Valášek. State derivative feedback by lqr for linear timeinvariant system. In Proc. of 16th IFAC World Congress, 2005. 
[4] C.E. Avellar and J.K. Hale. On the zeros of exponential polynomials. Mathematical analysis and applications, 73:434-452, 1980.

[5] R. Datko. Not all feedback stabilized hyperbolic systems are robust with respect to small time delays in their feedbacks. SIAM Journal on Control and Optimization, 26(3):697-713, 1988.

[6] R. Datko and Y.C. You. Some second-order vibrating systems cannot tolerate small time delays in their damping. Journal of Optimization Theory and Applications, 70:521-537, 1991.

[7] S.J. Dyke, B.F. Spencer, P. Quast, M.K. Sain, D.C. Kaspari, and T.T. Soong. Acceleration feedback control of MDOF structures. Journal of Engineering Mechanics, 122:907-917, 1996.

[8] J.K. Hale. Effects of delays on dynamics. In A. Granas, M. Frigon, and G. Sabidussi, editors, Topological methods in differential equations and inclusions, pages 191-238. Kluwer Academic Publishers, 1995.

[9] H. Kokame, K. Hirata, K. Konishi, and T. Mori. Difference feedback can stabilize uncertain steady states. IEEE Transactions on Automatic Control, 46(12):1908-1913, 2001.

[10] H. Logemann. Destabilizing effects of small time-delays on feedback-controlled descriptor systems. Linear Algebra and its Applications, 272:131-153, 1998.

[11] H. Logemann and R. Rebarber. The effect of small time-delays on the closed-loop stability of boundary control systems. Mathematics of Control, Signals and Systems, 9:123-151, 1996.

[12] H. Logemann and S. Townley. The effect of small delays in the feedback loop on the stability of neutral systems. Systems \& Control Letters, 27:267-274, 1996.

[13] W. Michiels, K. Engelborghs, D. Roose, and D. Dochain. Sensitivity to infinitesimal delays in neutral equations. SIAM Journal of Control and Optimization, 40:1134-1158, 2002.

[14] W. Michiels, S. Mondié, D. Roose, and M. Dambrine. The effect of approximating distributed delay control laws on stability. In Advances in Time-Delay Systems, Lecture Notes in Computational Science and Engineering, pages 207-225. Springer Verlag, 2004.

[15] W. Michiels and T. Vyhlídal. An eigenvalue based approach for the stablization of linear time-delay systems of neutral type. Automatica, 41:991-998, 2005. 
[16] S. Mondié and W. Michiels. Finite spectrum assignment of unstable time-delay systems with a safe implementation. IEEE Transactions on Automatic Control, 48(12):2207-2212, 2003.

[17] Ö. Morgül. On the stabilization and stability robustness against small delays of some damped wave equations. IEEE Transactions on Automatic Control, 40(9):1626-1630, 1995.

[18] S.-I. Niculescu. Delay effects on stability. A robust control approach, volume 269 of Lecture Notes in Control and Information Sciences. Springer Verlag, 2001.

[19] N. Olgac, H. Elmali, M. Hosek, and M. Renzulli. Active vibration control of distributed systems using delayed resonator with acceleration feedback. Transactions of ASME Journal of Dynamic Systems, Measurement and Control, 119, 1997.

[20] K. Pyragas. Continuous control of chaos by self-controlling feedback. Physics Letters A, 170:421-428, 1992.

[21] R. Rebarber and S. Townley. Robustness with respect to delays for exponential stability of distributed parameter systems. SIAM Journal on Control and Optimization, 37(1):230-244, 1999.

[22] R. Sipahi, G. Arslan, and S.-I. Niculescu. Some remarks on control strategies for continuous gradient play dynamics. In Proceedings of the 45th IEEE Conference on Decision and Control, San Diego, USA, 2006.

[23] G. Stépán. Retarded dynamical systems: stability and characteristic function, volume 210 of Research Notes in Mathematics. Longman Scientific: London, 1989.

\section{A A technical lemma}

Lemma A.1. Consider the equation

$$
\lambda-\mu\left(1-e^{-\lambda}\right)=0,
$$

where $\mu \in S$, with $S$ given by (12). Then all roots of (34) are in $\mathbb{C}^{-}$, except for a root at the origin with multiplicity one.

Proof. We distinguish two cases as below.

Case 1: $\mu \notin \mathbb{R}$.

We first characterize the zeros of the auxiliary function

$$
h(\lambda ; \nu):=\lambda-\mu\left(1-e^{-\lambda \nu}\right)
$$

as a function of the parameter $\nu \geq 0$. For $\nu=0$, the function $h$ has only one zero at the origin, which is invariant w.r.t. changes of $\nu$. If $\nu$ is increased from zero, then the number 
of zeros in the closed right half plane can only increase if zeros cross the imaginary axis. A zero at the origin with multiplicity larger than one cannot occur since

$$
h^{\prime}(0 ; \nu)=1-\mu \nu \neq 0 .
$$

If $h$ has a zero $j \omega, \omega \in \mathbb{R} \backslash\{0\}$, for some value of $\nu$, then we have:

$$
j \omega=\mu\left(1-e^{-j \omega \nu}\right) .
$$

Solving this equation yields:

$$
\omega=\omega^{*}:=2 \Im(\mu), \quad \nu=\nu_{k}^{*}:=\frac{\angle(\mu)+\pi k}{\Im(\nu)}, \quad k \geq 1 .
$$

Next, we look at the crossing direction of a zero on the imaginary axis w.r.t. the parameter $\nu$. Taking the derivative of $h(\lambda(\nu) ; \nu)=0$ w.r.t. $\nu$ at $(\lambda, \nu)=\left(j \omega^{*}, \nu_{k}^{*}\right)$ yields:

$$
\lambda^{\prime}\left(\nu^{*}\right)=\frac{j \omega^{*} \mu e^{-j \omega^{*} \nu^{*}}}{1-\nu \mu e^{-j \omega^{*} \nu}},
$$

from which we get

$$
\begin{aligned}
\Re\left(\lambda^{\prime}\left(\nu^{*}\right)^{-1}\right) & =\Re\left(\frac{1}{j \omega^{*} \mu e^{-j \omega^{*} \nu^{*}}}\right)=\Re\left(\frac{1}{j \omega^{*}\left(\mu-j \omega^{*}\right)}\right) \\
& =\Re\left((2 \Im(\mu)(\Im(\mu)+j \Re(\mu)))^{-1}\right)>0
\end{aligned}
$$

and

$$
\Re \lambda^{\prime}\left(\nu^{*}\right)>0 .
$$

From (35) and (36) we can conclude:

1. if $\nu \in\left[0, \nu_{1}^{*}\right)$, then all zeros of $h$ are in $\mathbb{C}^{-}$, except for a zero at the origin with multiplicity one;

2. if $\nu>\nu_{1}^{*}$, then $h$ has zeros in $\mathbb{C}^{+}$.

The assertion of the proposition is straightforward when taking into account that $\mu \in S$ $(\mu \notin \operatorname{clos}(S))$ is equivalent to $\nu_{1}^{*}>1\left(\nu_{1}^{*}<1\right)$.

Case 2: $\mu \in \mathbb{R}$.

The equation $h=0$ is the characteristic equation of the system $\dot{x}(t)=\mu x(t)-\mu x(t-\tau)$. The stability of this system has been analyzed in e.g. [18, 23], from which the statements of the proposition follow. 